\title{
Patient-Reported Quality of Hospital Discharge Transitions: Results from the SILVER-AMI Study
}

\author{
Bradley G. Richards, MD, MBA' , Alexandra M. Hajduk, PhD, MPH', Julia Perry, MD', \\ Harlan M. Krumholz, MD ${ }^{3,4,5}$, Ali M. Khan, MD, MPP ${ }^{5,6,7}$, and Sarwat I. Chaudhry, MD ${ }^{7}$
}

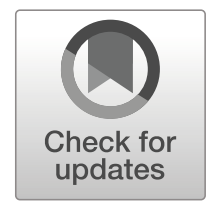

${ }^{1}$ Section of General Internal Medicine, Department of Internal Medicine, Yale School of Medicine, New Haven, CT, USA; ${ }^{2}$ Section of Geriatrics, Department of Internal Medicine, Yale School of Medicine, New Haven, CT, USA; ${ }^{3}$ Section of Cardiovascular Medicine, Department of Internal Medicine, Yale School of Medicine, New Haven, CT, USA; 'Department of Health Policy and Management, Yale School of Public Health, New Haven, CT, USA; ${ }^{5}$ Center for Outcomes Research and Evaluation, Yale-New Haven Hospital, New Haven, CT, USA; ${ }^{6}$ CareMore Health System, Cerritos, CA, USA; ${ }^{7}$ Department of Medicine, Pritzker School of Medicine, University of Chicago, Chicago, IL, USA.

BACKGROUND: Transitions from hospital to home in older patients are a high-risk period for adverse outcomes in a population that may have more challenges navigating the healthcare system. There is little information about the association of patientreported quality of hospital discharge processes with clinical outcomes.

OBJECTIVES: We evaluated whether patient-reported quality of hospital discharge processes was associated with emergency department utilization and rehospitalization within 30 days of discharge after hospitalization for acute myocardial infarction (AMI) in older adults.

DESIGN: Multi-center, prospective cohort study.

PATIENTS: The ComprehenSIVe Evaluation of Risk Factors in Older Patients with Acute Myocardial Infarction (SILVER-AMI) study was a longitudinal study of 3006 adults age 75 and older hospitalized with AMI recruited from 94 academic and community hospitals from across the USA.

INTERVENTION: N/A

MAIN MEASURES: Patients answered a subset of questions from the Hospital Consumer Assessment of Healthcare Providers and Systems (HCAHPS) survey. Readmissions and emergency department utilization within 30 days of discharge were ascertained through medical record review.

KEY RESULTS: A total of 2132 patients were included in the study. Patients' median age was 81 years and the response rate to the survey of discharge quality was $87 \%$. Patients who reported being asked about having the help they needed at home were significantly less likely to have emergency room utilization within 30 days of discharge in both the unadjusted $(0.65$, 95\% CI 0.43-0.99) and adjusted (0.65, 95\% CI 0.420.997) models, though there was no significant association with readmission.

CONCLUSION: Report of an assessment of help needed at home during hospitalization was associated with lower post-discharge emergency department utilization. Efforts to improve outcomes after hospital discharge in older patients may benefit from greater focus on assessing need of help at home.

Received May 13, 2019

Accepted September 23, 2019

Published online October 25, 2019
KEYWORDS: AMI; transitions; older adults; patient-reported quality; readmission.

$\mathrm{J}$ Gen Intern Med 35(3):808-14

DOI: $10.1007 / \mathrm{s} 11606-019-05414-8$

(C) Society of General Internal Medicine 2019

\section{INTRODUCTION}

Nearly 1 in 3 patients hospitalized for acute myocardial infarction (AMI) is aged 75 years or older and the incidence of coronary artery disease is expected to double in this age group by 2040. ${ }^{1,2}$ Older adults hospitalized for AMI are at significantly higher risk than younger patients for poor outcomes after hospital discharge, including rehospitalization for recurrent MI, heart failure, stroke, and death. ${ }^{3}$ Clinical risk factors and patient characteristics that predict readmission after AMI have been previously described. ${ }^{4-6}$ Another key component in reducing adverse outcomes such as hospital readmission and emergency department use after hospitalization for AMI is the successful transition to post-hospital care. After discharge, patients and their families assume a greater share of responsibility for ongoing healthcare. In the face of continuing recovery from acute illness, patients must learn to manage multiple new medications and navigate follow-up appointments, often with new medical providers. The successful execution of this discharge transition may be especially challenging for older patients given their higher burden of comorbid illness, as well as limited cognitive and physiologic reserve. ${ }^{7-9}$

While there is not a uniform agreement about the specifics of a high discharge quality, the major components include assessing and addressing post-hospital needs, medication reconciliation, patient education, and arranging post-hospital care follow-up. ${ }^{10,11}$ This requires good communication and care coordination efforts and working closely with patients and caregivers on post-discharge planning.

Despite the importance of the hospital discharge process, ongoing efforts to improve it, and the heightened complexities of the discharge transition in older patients, a rigorous understanding of patient-reported quality of the discharge transition and its impact on outcomes is notably lacking. ${ }^{12,}{ }^{13}$ This 
knowledge gap is particularly important because the Hospital Consumer Assessment of Healthcare Providers and Systems (HCAHPS) patient satisfaction survey is part of the Centers for Medicare and Medicaid Services (CMS) Hospital Value-Based Purchasing Program and survey responses have significant financial implications for hospitals and healthcare systems. ${ }^{14}$ The HCAHPS questionnaire is a survey instrument used by the US Centers for Medicare and Medicaid Services to measure patients' perceptions about recent hospital stays. ${ }^{15} \mathrm{CMS}$ has indicated that these scores represent quality from the patient experience perspective and will be a priority in CMS' efforts to improve patient care. ${ }^{16}$ Data remains limited on whether these HCAHPS survey questions are associated with clinical outcomes after hospital discharge for individual patients. ${ }^{17}$ The mandated use of these measures in the absence of robust data on their association with post-hospitalization clinical outcomes enhances the relevance of this knowledge gap.

Accordingly, the objective of this study was to evaluate whether older patients' reported quality of hospital discharge (based on HCAHPS questions) was associated with emergency department utilization and rehospitalization within 30 days of discharge. Data for this study comes from the ComprehenSIVe Evaluation of Risk Factors in Older Patients with Acute Myocardial Infarction (SILVER-AMI) study (NIH/NHLBI: 1R01HL115295), a prospective longitudinal study of 3041 adults aged 75 and older, hospitalized with AMI.

\section{METHODS}

\section{Data Source}

The Comprehensive Evaluation of Risk Factors in Older Patients Hospitalized for Acute Myocardial Infarction (SILVER-AMI) was an observational, prospective, cohort study of 3041 patients aged 75 years and older hospitalized for AMI who were recruited from 94 academic and community hospitals across the USA between January, 11, 2013, and October 20, 2016, with the last follow-up assessment completed on June 14, 2017. Details of the study have been published previously. ${ }^{18}$ Briefly, site coordinators identified hospitalized older adults with elevated cardiac biomarkers and reviewed medical records to confirm a diagnosis of AMI in accordance with the Third Universal Definition of Myocardial Infarction. ${ }^{19}$ Patients were excluded if they experienced AMI as the result of an inpatient procedure or surgery, were transferred from an outside hospital > $48 \mathrm{~h}$ after admission, or did not speak English or Spanish. Written informed consent was obtained from the patient (or a proxy in the instance of diminished capacity to consent (2.4\% of patients)). We obtained approval from the Institutional Review Boards at all study recruitment sites and at Yale University.

As part of the SILVER-AMI study, the 2417 patients discharged home were mailed a questionnaire to be completed 1 week after hospital discharge that referenced the index admission, which included 2 HCAHPS questions in the discharge information domain to assess their perceptions about quality of the discharge transition, as follows. "During this hospital stay, did doctors, nurses or other hospital staff talk with you about whether you would have the help you needed when you left the hospital? During this hospital stay, did you get information in writing about what symptoms or health problems to look out for after you left the hospital?" Questions were answered as either "Yes" or "No." Non-responders, 285 patients ( $11.8 \%$ of patients surveyed), were excluded from the analysis.

Readmissions and emergency department use were ascertained via medical record review, with patient-reported events (confirmed by medical record review) used to ensure completeness. During study enrollment, the patient reported the hospitals they used for medical care. When the 6-month follow-up window closed, the research coordinator contacted these identified hospitals to assess and collect readmission and emergency department records. Separately, as part of the 6-month follow-up interview, the patient also reported hospital readmissions to the Yale Coordinating Center. The Yale Coordinating Center then reconciled the hospital records collected by the coordinator against self-reported events to ensure that no readmissions were missing from the assessment. If necessary, we collected additional records to capture events that occurred at a hospital not initially indicated.

\section{Covariates}

We collected data on sociodemographic characteristics (age, sex, race, education, cohabitation status), comorbidities (prior

Association of Care Transition Quality Measures with Risk of ED Utilization at 30 Days

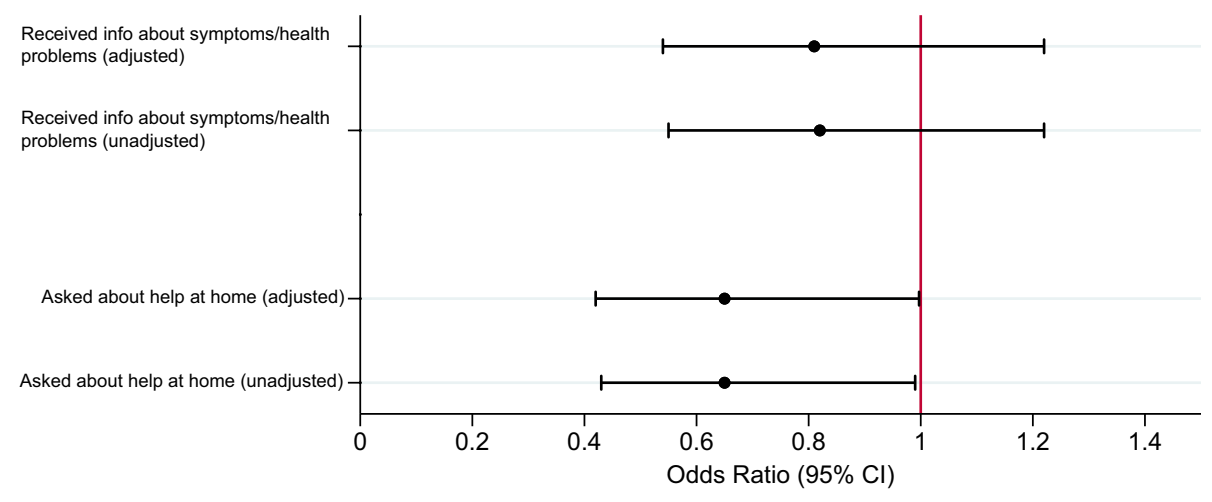

Figure 1 Association of HCAHPS measures with risk of ED utilization at 30 days. CI, confidence interval. 


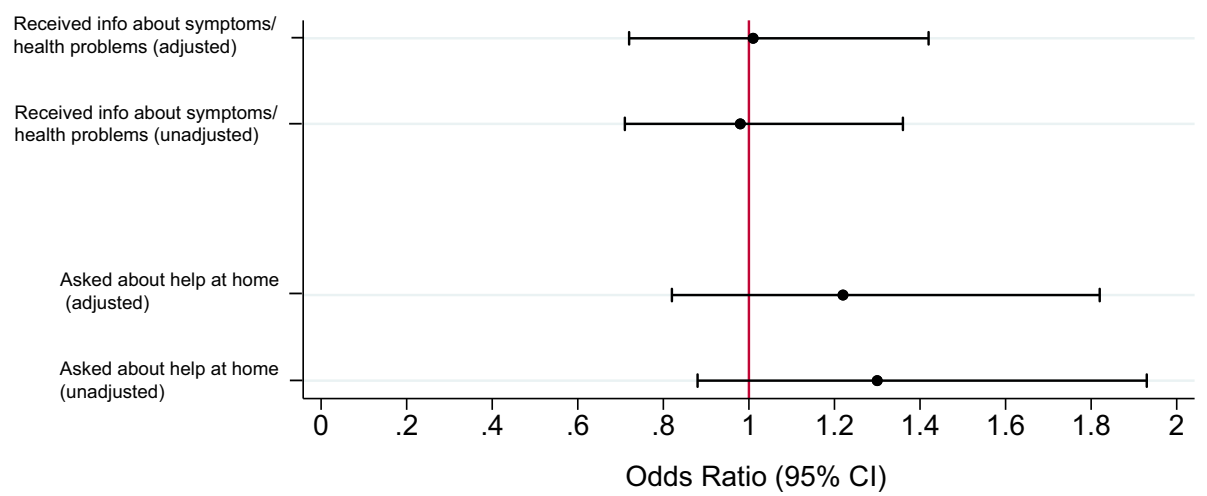

Figure 2 Association of HCAHPS measures with risk of rehospitalization at 30 days. CI, confidence interval.

CAD, AMI, revascularization, arrhythmia, or heart failure; peripheral vascular disease, valvular disease, $\mathrm{CVA}, \mathrm{COPD}$, chronic kidney disease, cancer, diabetes mellitus), and AMI and hospitalization characteristics (Killip class, presenting heart rate, presenting systolic blood pressure, time to presentation, MI classification [ST elevation versus non-ST elevation], length of admission, initial $\mathrm{Hb}$ value, in-hospital revascularization, in-hospital complications [arrhythmia, bleeding, heart failure, LVEF, and discharge location). ${ }^{20}$ The covariates that were collected from patient report include race, education, and cohabitation status. Comorbidities and AMI characteristics were obtained via medical record review.

\section{Analysis}

To describe the study population, we used means with standard deviations and proportions with confidence intervals (see Table 1). We used unadjusted and adjusted logistic regression analysis to evaluate associations between the dichotomous responses to each of the two discharge HCAHPS questions and subsequent healthcare utilization with the primary outcomes being emergency room utilization and hospital readmission within 30 days of discharge from the index hospitalization. The selection of variables for inclusion in the multivariable-adjusted model was guided by a literature review on determinants of hospital readmissions after AMI hospitalization (shown in Table 1). ${ }^{21,22}$ Findings were considered significant at $p$ value less than 0.05 in two-tailed tests. Missing data on covariates were accounted for via multiple imputation.

\section{RESULTS}

Of the 2417 patients discharged from the hospital to home, $2119(87.7 \%)$ responded to the question of whether a doctor, nurse, or other hospital staff member discussed whether they would have the help needed at home and 2111 (87.4\%) responded to whether they were given written information about symptoms or health problems to look out for. Nonrespondents tended to be older and were more often of nonwhite race. Sex and education did not vary significantly across respondents and non-respondents. The 285 patients missing data on both questions were excluded from the analysis.
Characteristics of the study sample are shown in Table 1 . The mean age of patients was 81.0 years (SD 4.8 years), $57.8 \%$ were male, $9.7 \%$ were non-white, $57.0 \%$ had less than a high school education, and $45.2 \%$ lived with their spouse or partner. The most common comorbidities were chronic kidney disease (CKD) (57.4\%), coronary artery disease (CAD) $(53.1 \%)$, and a prior history of cardiac revascularization $(42.2 \%) .59 .6 \%$ of patients

Table 1 Baseline Characteristics of Study Population $(N=2132)$

\begin{tabular}{ll}
\hline \hline Demographic & \\
Mean age (SD) & $81.0(4.8)$ \\
Male & $1232(57.8)$ \\
Non-white & $203(9.7)$ \\
Education $\leq 12$ years & $1205(57.0)$ \\
Married or living with partner & $963(45.2)$ \\
Comorbidities and prior medical history & \\
Chronic kidney disease [eGFR < 60 ml/min/1.73 m ${ }^{2}$ ] & $1223(57.4)$ \\
Coronary artery disease & $1131(53.1)$ \\
Revascularization & $900(42.2)$ \\
Diabetes mellitus & $752(35.3)$ \\
MI & $584(27.4)$ \\
Arrhythmias & $519(24.3)$ \\
Cancer & $453(21.3)$ \\
Congestive heart failure & $346(16.2)$ \\
Cerebrovascular disease & $285(13.4)$ \\
COPD & $269(12.6)$ \\
Peripheral arterial disease & $238(11.2)$ \\
Valvular heart disease & $227(10.7)$ \\
Presentation and hospitalization characteristics & \\
Median length of stay (IQR) & $3(2-6)$ \\
Mean heart rate [bpm] [first available] (SD) & $82.3(22.9)$ \\
Mean systolic BP [mmHg] [first available] (SD) II & $147.5(30.6)$ \\
Mean hemoglobin initial value [first available] (SD) & $13.0(2.0)$ \\
Killip class & \\
Killip I & $1901(89.2)$ \\
Killip I, II, III, or IV & $231(10.8)$ \\
Time from symptom onset to presentation & \\
< 6 h & $1263(59.6)$ \\
Z6 h & $857(40.4)$ \\
STEMI & $580(27.2)$ \\
Revascularization status & $253(11.9)$ \\
No cardiac catheterization & $363(17.0)$ \\
Cardiac catheterization without revascularization & $1344(63.0)$ \\
PCI only & $172(8.1)$ \\
CABG & \\
\hline
\end{tabular}

$S D$, standard deviation; eGFR, estimated glomerular filtration rate; $M I$, myocardial infarction; $C O P D$, chronic obstructive pulmonary disease; $I Q R$, interquartile range; BP, blood pressure; STEMI, ST-elevation myocardial infarction; $P C I$, percutaneous coronary intervention; $C A B G$, coronary artery bypass graft

Number of participants from which data was obtained presented with percentages of participants in parentheses, unless stated otherwise. All variables were missing $<5 \%$ of values 
presented within $6 \mathrm{~h}$ of AMI symptom onset, and the median length of stay was 3 days (IQR 2-6 days). Regarding coronary procedures during the AMI hospitalization, $63.0 \%$ underwent percutaneous coronary intervention (PCI), and $8.1 \%$ underwent coronary artery bypass grafting (CABG). $77.3 \%$ reported that they were asked about whether they would have help needed at home and $73.9 \%$ reported receiving written information about symptoms or health problems to look out for.

Considering 30-day outcomes, $202(8.4 \%)$ patients had at least one ED visit within 30 days of discharge and 410 (17.0\%) had at least one hospital readmission. In unadjusted analyses, patients who reported that they were asked about having the help they needed at home were less likely to have ED utilization within 30 days of discharge (OR 0.65 (95\% CI 0.43-0.99), $p=$ 0.043). After adjusting for all of the sociodemographic and clinical characteristics shown in Table 1, patients who reported being asked about help remained less likely to have ED utilization within 30 days of discharge (OR 0.65 (95\% CI 0.42 0.997 ), $p=0.049$ ) (Fig. 1 and Table 2). In unadjusted and adjusted analyses, report of receiving written information about symptoms or health problems to look out for after discharge was not significantly associated with ED utilization within 30 days of discharge (OR 0.82 (95\% CI 0.55-1.22) and OR 0.81 (95\% CI 0.54-1.22), respectively) (Table 3).] $\rightarrow$

Report of being asked about having the help needed at home had no significant association with readmission in both unadjusted and adjusted models (OR 1.304 (95\% CI 0.882 1.928 ) and OR 1.219 (95\% CI 0.818-1.815), respectively). Additionally, report of having received written information about symptoms or health problems to look out for after discharge had no significant association on 30-day readmission in unadjusted or adjusted models (OR 0.982 (95\% CI 0.709-1.359) and OR 1.012 (95\% CI 0.724-1.415), respectively) (Fig. 2).] $\rightarrow$

\section{DISCUSSION}

In a large, national cohort of older adults hospitalized for AMI, we found that report of being asked about help needed at home was associated with lower rate of postdischarge emergency department use, even after adjustment for factors known to be associated with clinical outcomes after AMI hospitalization. Notably, reported receipt of written information about health problems to

Table 2 Odds Ratios for Help You Need at Home. Association Between Report of Being Asked About Help at Home and ED Utilization and Readmission Within 30 Days of Discharge

\begin{tabular}{lll}
\hline \hline Outcome & $\begin{array}{l}\text { Unadjusted } \\
\text { OR (95\% CI) }\end{array}$ & $\begin{array}{l}\text { Multivariable-adjusted } \\
\text { OR (95\% CI) }\end{array}$ \\
\hline $\begin{array}{l}\text { Readmission within } \\
\text { 30 days }\end{array}$ & $1.304(0.882-1.928)$ & $1.219(0.818-1.815)$ \\
$\begin{array}{l}\text { Emergency room } \\
\text { visit within 30 days }\end{array}$ & $0.649(0.425-0.990)$ & $0.649(0.422-0.997)$ \\
\hline
\end{tabular}

OR, odds ratio; $C I$, confidence interval
Table 3 Odds Ratios for Writen Information. Association Between Report of Having Received Written Information About Symptoms or Health Problems to Look out for After Discharge and ED Utilization and Readmission Within 30 Days of Discharge

\begin{tabular}{lll}
\hline \hline Outcome & $\begin{array}{l}\text { Unadjusted OR } \\
\mathbf{( 9 5 \%} \mathbf{C I})\end{array}$ & $\begin{array}{l}\text { Multivariable- } \\
\text { adjusted OR (95\% CI) }\end{array}$ \\
\hline $\begin{array}{l}\text { Readmission within } \\
\text { 30 days }\end{array}$ & $\begin{array}{l}0.982(0.709- \\
1.359)\end{array}$ & $1.012(0.724-1.415)$ \\
$\begin{array}{l}\text { Emergency room visit } \\
\text { within 30 days }\end{array}$ & $\begin{array}{l}0.815(0.545- \\
1.219)\end{array}$ & $0.810(0.537-1.221)$ \\
\hline
\end{tabular}

OR, odds ratio; $C I$, confidence interval

look out for after discharge had no significant impact on ED utilization or readmissions. There has been great focus on the discharge transition, yet limited evidence to date to support the association between patient-reported quality of the transition and clinical outcomes. Our findings provide support for assessing help needed at home in older patients prior to hospital discharge.

There are several possible explanations for our observations. It is increasingly recognized that patients leave the hospital in a state of heightened vulnerability, not only due to their acute illness, but also due to the experience of hospitalization itself. Sleep deprivation, limited mobility, poor nutrition, and psychological stress may diminish patients' capacity for self-care after discharge. ${ }^{23}$ Notably, self-care requirements after AMI hospitalization are numerous. The term "treatment burden" has been used to describe the numerous tasks patients and caregivers must execute on a daily basis to manage their health conditions, ${ }^{24}$ and after AMI hospitalization, treatment burden can be high, with new dietary restrictions and physical activity recommendations, changes to medications, and need for follow-up appointments and diagnostic testing. In light of this high treatment burden and patient's diminished capacity for self-care after hospitalization, it is perhaps unsurprising that talking with patients about help needed at home is more important in reducing avoidable healthcare utilization than instructing them on problems they might encounter.

Our study extends prior literature on patient-reported quality of hospital discharge transitions. A recent study by Goldstein et al. examined the association between patientreported discharge quality and 30-day hospital readmissions among patients undergoing PCI or CABG. ${ }^{25}$ In this study, patient-reported discharge quality independently influenced readmission rates; better quality was associated with lower readmission rates. An important consideration is that this study used the Care Transitions Measure-3 (CTM-3) to assess discharge quality, a component of the HCAHPS assessment. Notably, the CTM-3 has been called into question because of psychometric inconsistencies. ${ }^{26}$ Brown et al. examined responses to the same HCAHPS questions used in our study among patients hospitalized for AMI. An affirmative response to both HCAHPS questions was used to indicate high hospital quality. They found that higher quality hospitals had lower 
readmission rates. ${ }^{27}$ Hachem et al. examined several HCAHPS questions, including the two used in our study, to determine their relationship with readmissions among 30,968 adult patients discharged from 10 hospitals with any diagnosis. They found that report of being asked about help at home was associated with increased risk of readmissions, while report of having written discharge instructions did not impact readmissions. ${ }^{28}$ The paradoxical finding of an increased readmission risk associated with being asked about help at home was thought to be explained by patients with greater illness severity being more likely to be asked about help at home. Both of these studies using HCAHPS questions (Brown et al. and Hachem et al.) relied upon hospital-reported responses either directly or from CMS, which has an average response rate of $27 \%$, raising considerable concerns about non-response bias. ${ }^{29}$ In contrast, the response rate to HCAHPS questions in our study was $87.7 \%$. Additionally, we examined emergency department utilization in addition to readmission rates, providing a fuller picture of the effects of patient-reported transition quality.

It is notable that neither of the HCAPHS measures for patient-reported quality of discharge transitions was associated with readmission in our study. It is possible that older adults have clinical complexity-including multimorbid chronic diseases, functional impairments, and psychosocial challenges - that makes many readmissions after AMI hospitalization unavoidable, even with optimal discharge processes of care. ${ }^{30}$ Illustrating their tenuous condition, among Medicare patients admitted for heart failure, pneumonia, or COPD, more than half of readmissions are for primary diagnoses different from the initial admission. ${ }^{31}$ Additionally, factors related to the healthcare system have been shown to strongly influence readmission rates with Medicare enrollees being more likely to be readmitted if they were discharged from the lowest quartile hospitals compared with individuals discharged from the highest quartile hospitals in regard to readmission performance. ${ }^{32}$ These patient-level and system-level factors that influence readmission rates may overshadow the effects of patient-reported quality of the discharge transition.

There is evidence that patient-reported quality of discharge documentation is not associated with readmissions, and our findings provide further evidence that written documentation about problems that may be encountered at home is insufficient for reducing post-discharge healthcare utilization. ${ }^{33,34}$ Given the significant cost both in administering HCAHPS and the impact responses can have for hospital payments, ensuring that each question adds value is important in improving clinical outcomes, patient satisfaction, and minimizing regulatory burden. Our findings suggest that the questions included in hospital quality assessments may need to be reconsidered, at least in the context of reducing 30-day healthcare utilization.

Our study had several notable strengths. First, the prospective nature of our study allowed for detailed and extensive data collection of demographics, comorbidities, presentation characteristics, and treatment, which we used to rigorously adjust for potential confounders in multivariable models. These models allowed us to isolate the effect of discharge quality on readmission and emergency department utilization, as known factors that influence post-discharge utilization were adjusted for. An additional strength of this study includes the recruitment of patients from a national network of hospitals across the USA, including academic, community, urban, and rural areas. This sampling enhances the external generalizability of our findings. Finally, the response rate for the survey of discharge quality was nearly $90 \%$, which further enhances the generalizability of our findings, since they were minimally impacted by non-response bias, which is an important limitation of studies that rely upon HCAHPS data provided by CMS or healthcare systems.

There are several issues which need to be considered in the interpretation of our findings. Patients completed the survey after returning home, introducing the possibility of recall bias. We did a robust review with patients or proxies of their healthcare utilization in the 6 months after the index admission. To complement self-reported utilization, medical records were obtained from all hospitals identified by participants as usual sources of care. However, it is possible that patients utilized healthcare at hospitals they did not identify to study staff, which may have led to underreporting of utilization. We only examined whether help needed was assessed and written instructions were provided, but we did not assess the quality of these assessments and instructions. Additionally, the two HCAHPS questions used do not capture all of the aspects of perceived discharge quality. We did not collect data to indicate which clinical team member discussed whether patients would have help needed at home, nor did we assess whether unmet needs were subsequently addressed.

The educational attainment in our study is lower than average for the older populations in the USA, raising concern for generalizability. ${ }^{35}$ Of note, rates of AMI and cardiovascular disease are notably higher among individuals with low educational attainment making this an important demographic to further understand. ${ }^{36,37}$ Finally, this study focused on older patients admitted with AMI; generalizability to younger, nonAMI populations is uncertain. However, given the high rates of AMI hospitalization among older patients, our findings have high clinical relevance even if only applied to older AMI populations.

Future studies should evaluate whether assessing and addressing unmet needs at home upon discharge improves post-discharge outcomes. Additionally, further studies are needed to determine which interventions are most effective and can be used to address unmet needs in a costefficient manner. ${ }^{38}$ With the shift to value-based payments, more funding will be available to address these unmet needs. There will likely be growing interest in costeffective interventions, such as incorporating Community 
Health Workers (CHWs) in the discharge process, which has demonstrated some limited success at improving care for patients with high post-hospitalization healthcare utilization. $^{39,} 40$

In summary, this study demonstrates a significant relationship between patient-reported assessment of help needed at home and 30-day ED utilization in older patients discharged after AMI hospitalization.

The growing population of older AMI patients coupled with their heightened vulnerability and treatment burden after AMI hospitalization highlights the importance of understanding and improving care transitions in this population. Ensuring that patients are equipped with the help needed may be a useful strategy in improving outcomes after hospital discharge.

Corresponding Author: Bradley G. Richards, MD, MBA; Section of General Internal Medicine, Department of Internal MedicineYale School of Medicine, New Haven, CT, USA (e-mail: Brad. Richards@yale.edu).

Funding Information This research was financially supported by the National Heart, Lung, and Blood Institute of the National Institutes of Health (RO1HL115295).

\section{Compliance with Ethical Standards:}

Written informed consent was obtained from the patient (or a proxy in the instance of diminished capacity to consent (2.4\% of patients)). We obtained approval from the Institutional Review Boards at all study recruitment sites and at Yale University.

Conflict of Interest: Dr. Krumholz reports personal fees from UnitedHealth, ownership (with spouse) of Hugo, personal fees from IBM Watson Health, personal fees from Element Science, personal fees from Aetna, contracts from the Centers for Medicare \& Medicaid Services, grants from Medtronic and the Food and Drug Administration, grants from Medtronic and Johnson and Johnson, personal fees from Arnold \& Porter, personal fees from Ben C. Martin Law Firm, personal fees from Facebook, grants from Shenzhen Center for Health Information, personal fees from National Center for Cardiovascular Diseases, Beijing, outside the submitted work. Dr. Khan reports personal fees and other from Anthem, Inc., other from Iora Health, outside the submitted work. Dr. Chaudhry serves as a reviewer for the CVS Caremark State of Connecticut Clinical Program. All remaining authors declare that they do not have a conflict of interest.

Disclaimer: The content is solely the responsibility of the authors and does not necessarily represent the official views of the National Institutes of health.

\section{REFERENCES}

1. Anderson JL, Adams CD, Antman EM, et al. 2011 ACCF/AHA Focused Update Incorporated Into the ACC/AHA 2007 Guidelines for the Management of Patients With Unstable Angina/Non-ST-Elevation Myocardial Infarction: a report of the American College of Cardiology Foundation/American Heart Association Task Force on Practice Guidelines. Circulation. 2011;123:e426-579.

2. Ho PM, Eng MH, Rumsfeld JS, et al. The influence of age on health status outcomes after acute myocardial infarction. Am Heart J 2008; 155:855-61.

3. Benjamin EJ, Blaha MJ, Chiuve SE, et al. Heart Disease and Stroke Statistics-2017 Update: A Report From the American Heart Association. Circulation. 2017;135:e146-603.

4. Brown JR, Conley SM, Niles NW. Predicting Readmission or Death After Acute ST-Elevation Myocardial Infarction. Clin Cardiol 2013;36:570-5.
5. Khawaja FJ, Shah ND, Lennon RJ, et al. Factors associated with 30day readmission rates after percutaneous coronary intervention. Arch Intern Med 2012;172:112-7

6. Hannan EL, Zhong $\mathbf{Y}$, Krumholz $\mathbf{H}$, et al. 30-day readmission for patients undergoing percutaneous coronary interventions in New York state. JACC Cardiovasc Interv 2011;4(12):1335-42.

7. Coleman EA, Berenson RA. Lost in transition: challenges and opportunities for improving the quality of transitional care. Ann Intern Med 2004;141:533-6.

8. Greysen SR, Hoi-Cheung D, Garcia V, et al. "Missing pieces"-functional, social, and environmental barriers to recovery for vulnerable older adults transitioning from hospital to home. J Am Geriatr Soc 2014;62:1556-61.

9. Albrecht JS, Gruber-Baldini AL, Hirshon JM, et al. Hospital discharge instructions: comprehension and compliance among older adults. J Gen Intern Med 2014;29:1491-8.

10. Transitions of Care: Technical Series on Safer Primary Care. Geneva: World Health Organization; 2016. Licence: CC BY-NC-SA 3.0 IGO.

11. Verhaegh KJ, MacNeil-Vroomen JL, Eslami S, Geerlings SE, de Rooij SE, Buurman BM. Transitional care interventions prevent hospital readmissions for adults with chronic illnesses. Health Aff 2014;33:1531-9.

12. Horwitz LI, Moriarty JP, Chen C, et al. Quality of discharge practices and patient understanding at an academic medical center. JAMA Intern Med 2013;173:1715-22.

13. Horstman MJ, Mills WL, Herman LI, et al. Patient experience with discharge instructions in postdischarge recovery: a qualitative study. BMJ Open 2017;7:e014842.

14. Giordano LA, Elliott MN, Goldstein E, Lehrman WG, Spencer PA. Development, implementation, and public reporting of the HCAHPS survey. Med Care Res Rev 2010;67:27-37.

15. Centers for Medicare \& Medicaid Services. Available at: https://www. hcahpsonline.org. Accessed 6 November 2018.

16. Federal Register. Department of Health and Human Services: Center for Medicare and Medicaid Services. Vol. 80 no 158 2015, p 49550.

17. Isaac T, Zaslavsky AM, Cleary PD, Landon BE. The relationship between patients' perception of care and measures of hospital quality and safety. Health Serv Res 2010;45:1024-40.

18. Dodson JA, Geda M, Krumholz HM, et al. Design and rationale of the comprehensive evaluation of risk factors in older patients with AMI (SILVER-AMI) study. BMC Health Serv Res 2014;14:506.

19. Thygesen, K. Alpert JS, Jaffe AS, Simoons ML, Chaitman BR, White HD. Third universal definition of myocardial infarction. Eur Heart J, 2012;33:2551-67.

20. Killip T, Kimball JT. Treatment of myocardial infarction in a coronary care unit: a two year experience with 250 patients. Am J Cardiol 1967;20:457-64.

21. Mendes de Leon CF, Rajan KB. Psychosocial influences in onset and progression of late life disability. J Gerontol B Psychol Sci Soc Sci. 2014;69:287-302

22. Barnes DE, Mehta KM, Boscardin WJ, et al. Prediction of recovery, dependence or death in elders who become disabled during hospitalization. J Gen Intern Med 2013;28:261-8.

23. Krumholz HM. Post-hospital syndrome-an acquired, transient condition of generalized risk. N Engl J Med. 2013;368:100-2.

24. Ridgeway $\mathbf{J L}$, Egginton JS, Tiedje $\mathbf{K}$, et al. Factors that lessen the burden of treatment in complex patients with chronic conditions: a qualitative study. Patient Prefer Adherence 2014;8:339.

25. Goldstein JN, Hicks LS, Kolm P, Weintraub WS, Elliott DJ. Is the Care Transitions Measure Associated with Readmission Risk? Analysis from a Single Academic Center. J Gen Intern Med 2016;31:732-8.

26. Anatchkova MD, Barysauskas CM, Kinney RL, et al. Psychometric evaluation of the Care Transition Measure in TRACE-CORE: do we need a better measure? J Am Heart Assoc 2014;3:e001053.

27. Brown JR, Chang CH, Zhou W, Mackenzie TA, Malenka DJ, Goodman DC. Health system characteristics and rates of readmission after acute myocardial infarction in the United States. J Am Heart Assoc 2014;3:e000714.

28. Hachem F, Canar J, Fullam MA, Andrew S, Hohmann S, Johnson C. The relationships between HCAHPS communication and discharge satisfaction items and hospital readmissions. PXJ 2014;1:71-77.

29. Centers for Medicare \& Medicaid Services. Available at: https://www. hcahpsonline.org. Accessed 1 April 2019.

30. Raval AD, Zhou S, Wei W, Bhattacharjee S, Miao R, Sambamoorthi U. 30-Day Readmission Among Elderly Medicare Beneficiaries with Type 2 Diabetes. Popul Health Manag 2015;18:256-64. 
31. Jencks SF, Williams MV, Coleman EA. Rehospitalizations among patients in the Medicare fee-for-service program. N Engl J Med 2009;360: 1418-28.

32. Krumholz HM, Wang $\mathbf{K}$, Lin Z, et al. Hospital-Readmission Risk - Isolating Hospital Effects from Patient Effects. N Engl J Med 2017;377:1055-64.

33. Jha AK, John Orav E, Epstein AM. Public Reporting of Discharge Planning and Rates of Readmissions. N Engl J Med 2009;361:2637-45.

34. Hansen LO, Strater A, Smith L, et al. Hospital discharge documentation and risk of rehospitalisation. BMJ Qual Saf 2011;20:773-8.

35. U.S. Department of Health and Human Services (USDHHS), Administration on Aging, Administration for Community Living. A Profile of Older Americans: 2018. Available at https://acl.gov/sites/default/files/ A ging \% 20 and \% 20 Dis ability\% 20 in \% 20 America / 2018OlderAmericansProfile.pdf. Accessed 25 July 2019.

36. Mackenbach JP, Stirbu I, Roskam AJ, et al. Socioeconomic inequalities in health in 22 European countries. N Engl J Med 2008;358:2468-81.
37. Igland J, Vollset SE, Nygård OK, Sulo G, Ebbing M, Tell GS. Educational inequalities in acute myocardial infarction incidence in Norway: a nationwide cohort study. PLoS One 2014;9:e106898.

38. Hansen LO, Young RS, Hinami K, Leung A, Williams MV. Interventions to reduce 30-day rehospitalization: a systematic review. Ann Intern Med $2011 ; 155: 520-8$

39. Landers S, Levinson M. Mounting Evidence of the Effectiveness and Versatility of Community Health Workers. Am J Public Health 2016;106;591-2.

40. Bailey JE, Surbhi S, Bell PC, Jones AM, Rashed S, Ugwueke MO. SafeMed: using pharmacy technicians in a novel role as community health workers to improve transitions of care. J Am Pharm Assoc 2016;56:73-81.

Publisher's Note Springer Nature remains neutral with regard to jurisdictional claims in published maps and institutional affiliations. 